\title{
Subminiature Multi-stage Band-Pass Filter Based on LTCC Technology Research
}

\author{
Bowen Li and Yongsheng Dai
}

\begin{abstract}
A subminiature multi-stage band-pass filter method, which is based on LTCC technology, was studied. The band-pass filter is composed of four good-performance resonator, among which transmission zeros achieved good stopband attenuation through cross coupling. Circuit simulation and the 3D electromagnetic field simulation were used to optimize the simulation. Agreements were found between the experiment results and simulation results. The centre frequency of the four-order band-pass filter is $5.25 \mathrm{GHz}$ and the bandwidth is 500MHz. Insertion loss is over $2.85 \mathrm{~dB}$ within the scope of pass-band, in the frequency ranges of $0-4.8 \mathrm{GHz}$ and above $6.3 \mathrm{GHz}$, the out-of-band attenuation is higher than $40 \mathrm{~dB}$. Additionally, the size is only $2.5 \mathrm{~mm} \times 3.2 \mathrm{~mm} \times 1.5 \mathrm{~mm}$. The filter range belongs to $\mathrm{C}$ spectrum with short wavelength. Hence, the stripline distributed structure was adopted to achieve the miniaturization of the filter.
\end{abstract}

Index Terms-Band-pass filter, LTCC, strip line, transmission zeros.

\section{INTRODUCTION}

With the significant development in the information industry and the radio communication system, microwave spectrum becomes more crowded than ever, the division of frequency band resource turns to be more delicate, and the frequency interval assigned to all kinds of communication system is getting closer. As a result, it leads to a higher requirement for the filter's electrical performance and comprehensive performance of miniaturization, integration, stability etc. In order to reduce losses and miniaturize the device to acquire higher quality factor, new materials and technology are needed. Due to the factors that small in size, good three-dimensional integrated flexibility, favourable ceramic material, and simple manufacturing process, the low temperature co-firing ceramic (LTCC) technology has become a research hotspot in the field of microwave.

LTCC band-pass filter is a high frequency ceramic microwave filter that is processed through electronic ceramic material stretch forming process, laminated in low temperature sintering technology, high precision printing laminated technology and packaging technology, and many other process. It is featured with high frequency, small volume, little insertion loss, and out-of-band attenuation [1].

LTCC technology has many advantages comparing to traditional packaging integration technology [2]: (1) easy to implement more wiring layer to improve the density of

Manuscript received October 10, 2015; revised January 13, 2016.

The authors are with the School of Electronic and Optical Engineering, Nanjing University of Science and Technology, Nanjing, China (e-mail 734955857@qq.com, daiyongsheng1005@126.com). package; (2) easy to be embedded within the components, which makes multi-function possible; (3) it allows to facilitate quality inspection on each layer wiring and interconnect hole before firing junction plate, which is conducive to improve the yield and quality of multi-layer substrate, shorten the production cycle, and reduce the cost; (4) good frequency characteristic and high speed transmission characteristic; (5) easy to form different structures of cavity, which results in good performance of the multi-functional microwave MEC; (6) easy to implement multi-layer wiring and encapsulation structure integration, which leads to further volume and weight reduction, and improving reliability. Because of its unique advantages, great superiority will be found using LTCC technology for surface assembly components in the new generation of mobile communication.

The main advantage of LC structure filter is the miniaturized volume. However, the structure design is more complicated and the attenuation tends to be larger. While the strip line LTCC filter resonance unit, which is composed of a transmission line, is suitable for the $\mathrm{C}$ band within high frequency band with the advantages of small volume, light weight, good stability, ease to connect with other microwave components. Hence, it is widely used in microwave integrated circuits.

This article studied strip line LTCC technology based sub-miniature band-pass filter that is designed with the strip line distributed structure [3]. Specifications of the filter are listed as follows: (1) the filter centre frequency is $5.25 \mathrm{GHz}$ with the bandwidth of $500 \mathrm{MHZ}$; (2) insertion loss in-band is less than $2.85 \mathrm{~dB}$, while the out-band restraint is over $40 \mathrm{~dB}(0$ $\mathrm{GHz}<f<4.8 \mathrm{GHz}$ and $6.3 \mathrm{GHz}<f$ ); (3) voltage standing wave ratio is 1.69 or less; (4) transmission zeros in the filter is designed by introducing cross coupling, which makes it is easy to implement; (5) introduction of cross coupling between the first and the fourth resonator makes it more attenuation in the stop-band; (6) the filter size is $2.5 \mathrm{~mm} \times 3.2 \mathrm{~mm} \times 1.5 \mathrm{~mm}$.

\section{THE THEORETICAL ANALYSIS}

\section{A. Theory}

Four-order strip line distributed structure band-pass filter equivalent circuit diagram is shown in Fig. 1. It is based on band-pass filter of four-order coupled resonator.

The computing formulas for inductance and capacitance and coupling coefficient of four-order coupled resonator are demonstrated in (1)-(4), in which $w$ is the width of the strip line; $d$ is the distance between two adjacent strip line; $b$ is the distance between the strip line and the ground; $l$ is the length of the strip line; $f_{1}$ and $f_{2}$ are two Eigen frequencies; $\mu$ is the 
four resonator magnetic permeability; $\varepsilon$ is the dielectric constant in homogeneous medium [4], [5].

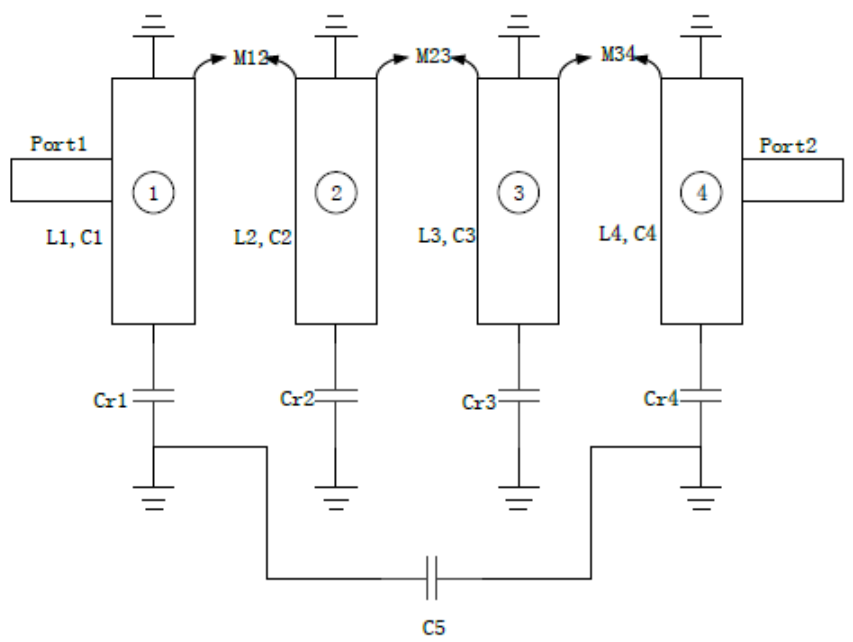

Fig. 1. Four-order coupled resonator equivalent circuit.

$$
\begin{gathered}
L_{i}=\frac{l \pi \mu}{8 \operatorname{arch}(\exp (\pi w / 2 b))}(H) \\
C_{i}=8 l \varepsilon \operatorname{arch}(\exp (\pi w / 2 b))(F) \\
C r_{i}=\frac{l \varepsilon w}{d}(F) \\
M_{i j}=\frac{f_{1}^{2}-f_{2}^{2}}{f_{1}^{2}+f_{2}^{2}}
\end{gathered}
$$

\section{B. The Principle of Zero}

In terms of band-pass filter, when strong signal needs to be inhibited or suppress range centre frequency is close to the mirror frequency, stop-band attenuation becomes insufficient [6]. As a consequence, transmission zeros are inserted on the suppression frequency for better inhibition effect. In microwave circuits, there are varies types of transmission zeros, which are (1) microwave signal going through different pathways to form the reversed offset; (2) going through a series of resonance signal into ground and reflected by the main parallel resonator etc.; (3) all distributed filter. The distributed filter method was used for this study. The four-order resonator phase change is shown in Fig. 2.

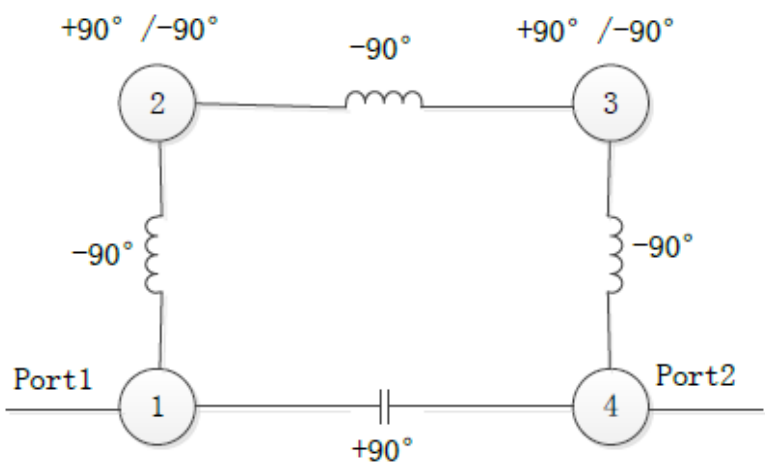

Fig. 2. The Four-order resonator phase change characteristics.
According to the Cross-Coupling Theory: when the signal goes through the first resonator, the main energy is transferred to the second and the third resonators, and the rest of the microwave signal is transferred to the fourth resonator; then the energy is also transmitted to the second resonator and the fourth resonator through cross coupling. The phase shift of magnetic coupling is $-90^{\circ}$, which the phase shift of electric coupling is $+90^{\circ}$; the phase shift through the resonator at the resonance frequency point is $0^{\circ}$; the phase shift when the frequency is below the resonance frequency is $+90^{\circ}$, while the signal phase shift is $-90^{\circ}$ when the frequency is higher than the resonant frequency; for those signals whose frequencies are lower than the resonant frequency, the main phases are: $-90^{\circ}$ $+90^{\circ}-90^{\circ}+90^{\circ}-90^{\circ}=-90^{\circ}$; the cross-phase is $90^{\circ}$. Hence, two signals reversed phase offset formed zero. For those whose frequencies are higher than the resonant frequency, the main phases are: $-90^{\circ}-90^{\circ}-90^{\circ}-90^{\circ}-90^{\circ}=-90^{\circ}$; the main phase is $90^{\circ}$. Hence, two signals offset formed zero. As a result, there is a zero on each side of the passband in this structure [7], [8].

\section{THREE DIMENSIONAL REALIZATION WAY}

\section{A. The Design Methodology and Process}

First of all, the series and the circuit topology were determined according to indicators of the filter; after which the size of the resonator was calculated based on the solution of intrinsic mode and the theory of dual mode coupled with the help of HFSS simulation software; then adjacent resonator coupling distance and the coupling coefficient is determined. Secondly, the coupling capacitance was adjusted to control the position of transmission zeros, which resulted in a good damping effect; the experimental design was used for debugging, filter designing, analysis and performance test [9].

\section{B. The Three-Dimensional Structure of Four-Order Filter}

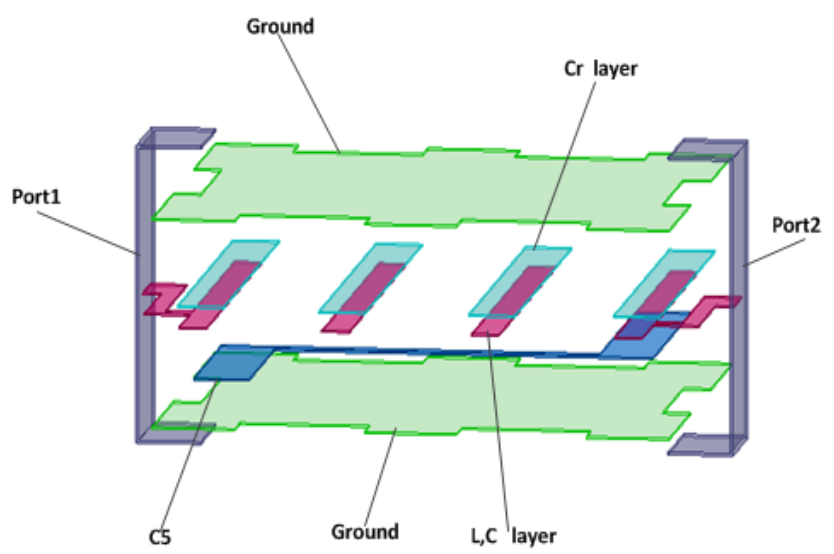

Fig. 3. The four-order filter internal three-dimensional structure.

The center frequency of this design is $5.25 \mathrm{GHz}$, which belongs to the $\mathrm{C}$ band with high frequency and short wavelength. Based on three-dimensional model of LTCC technology, the volume of the filter is $2.5 \mathrm{~mm} \times 3.2 \mathrm{~mm} \times 1.5$ $\mathrm{mm}$; the medium is ceramic materials whose relatively dielectric constant is 7.6 and the dielectric loss angle is 0.0013; the metal conductor material is silver with the thickness of $0.01 \mathrm{~mm}$. As shown in Fig. 3, 3D model consists 
five layers, among which the first and the fifth layer is the ground plane; the second is the load capacitance layer; while the third is the LC layer; and the second and the third layers are parallel, which constitutes four-order resonance unit; the fourth layer is cross-coupling capacitance between the first and the fourth resonator [10].

\section{THE RESUlts OF SimUlation AND TEST}

The overall design was completed with reference to the filter parameters; producing and processing were completed as well. The production samples and test fixture are illustrated in Fig. 4 and Fig. 5, respectively.

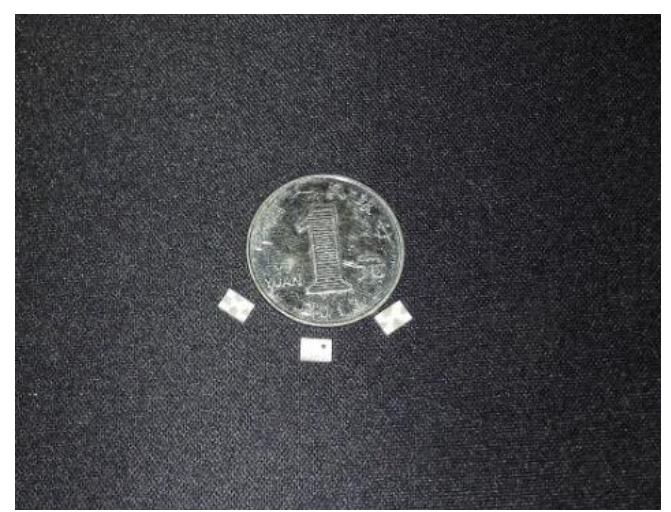

Fig. 4. The four-order band-pass filter real figure.

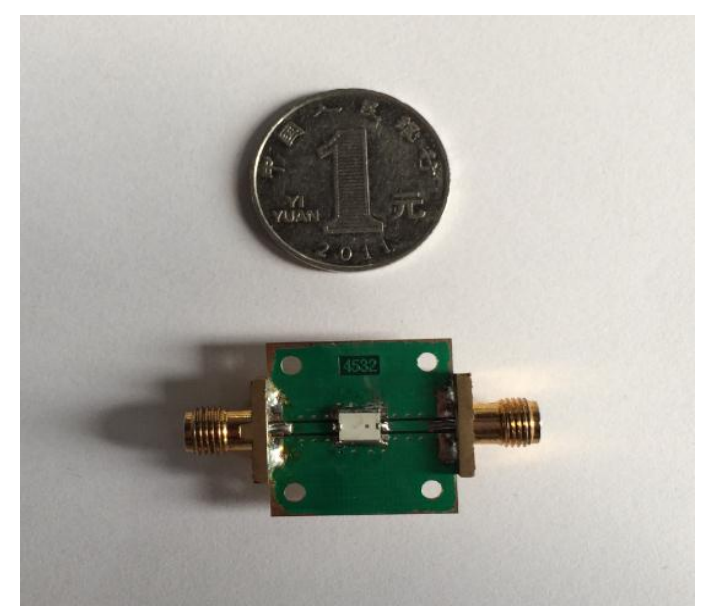

Fig. 5. The four-order band-pass filter test fixture.

The simulation results of four-order band-pass filter are demonstrated in Fig. 6; from which the following results can be concluded: the centre frequency is $5.25 \mathrm{GHz}$; the band-width is $500 \mathrm{MHz}$; the in-band insertion loss is less than $1.59 \mathrm{~dB}$ and out-band restraint is more than $40 \mathrm{~dB}(0 \mathrm{GHz}<f$ $<4.65 \mathrm{GHz}$ and $5.9 \mathrm{GHz}<f$ ); standing wave is larger than $24 \mathrm{~dB}$.

The experimental result of four-order band-pass filter is shown Fig. 7. It can be read from the result that the in-band insertion loss is $2.85 \mathrm{~dB}$, while the out-band restraint is more than $40 \mathrm{~dB}(0 \mathrm{GHz}<f<4.8 \mathrm{GHz}, 6.3 \mathrm{GHz}<f)$; the voltage standing wave ratio no larger than 1.69. Though slight difference could be found between experimental and simulation results, the difference tends to be consistent; moreover, the results also meet the requirements of this design.

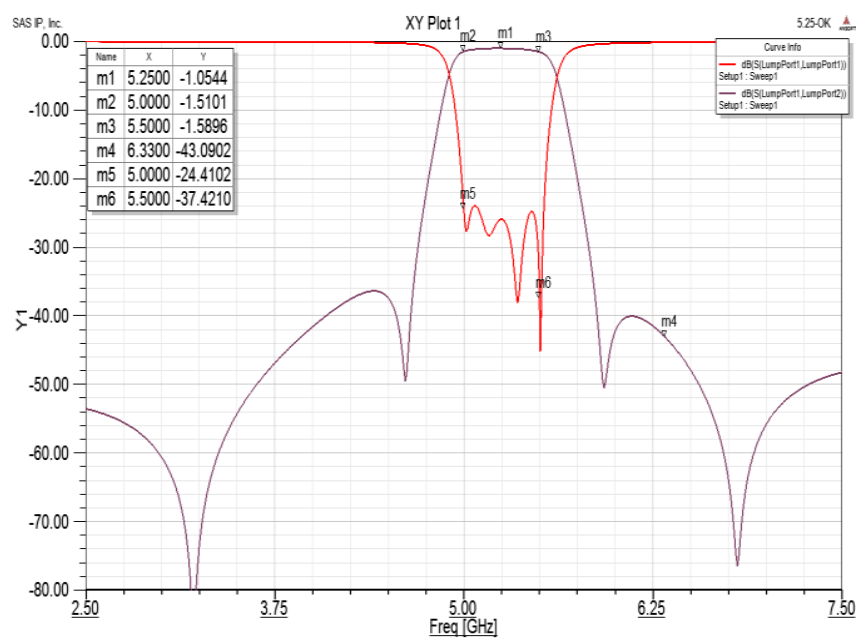

Fig. 6. The four-order filter simulation result.

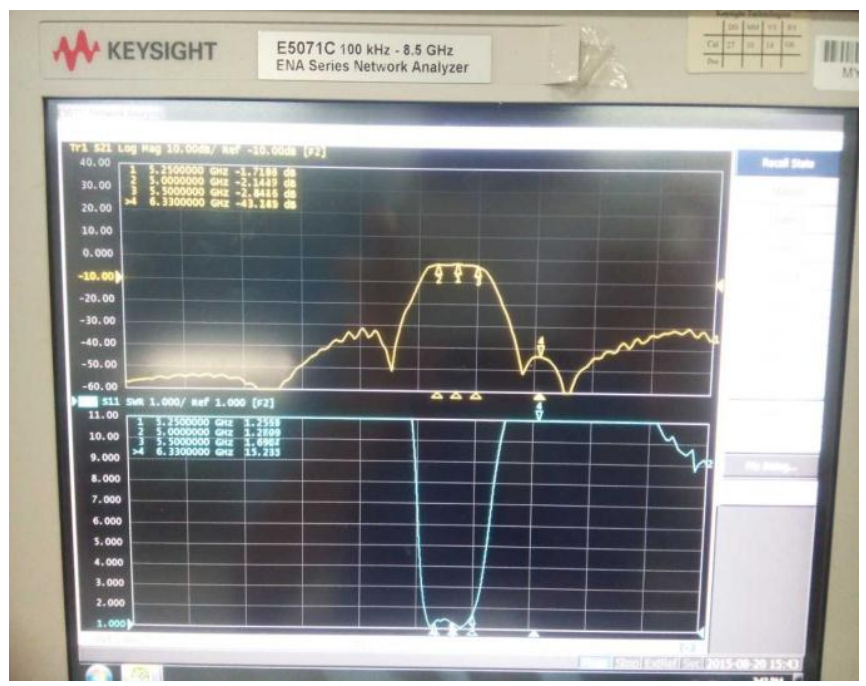

Fig. 1. The four-order band-pass filter material test pattern.

\section{CONCLUSION}

A series of simulation test, production and processing work have been carried out based on the design scheme. From the results, it can be concluded that two transmission zeros provides good performance, which is good stop-band attenuation and steep side-band. Circuit simulation and 3D simulation software were used for filter design analysis and the optimization simulation. Good agreement was found between the experimental and simulating results; and the results met the design requirements. Furthermore, the filter structure is simple and easy to manufacture; hence, it is suitable for batch process, defense precision electronic equipment, and radio frequency wireless communication system. Further research may be including more stages filter, such as five-order filter and six-order filter, compared to the four-order filter, summarize the rules.

\section{REFERENCES}

[1] M. F. Xiang, "Subminiature high-performance LTCC microwave filter design and research," Nanjing University of Science and Technology, 2007.

[2] Y. S. Dai, X. X. Tang, W. K. Zhou et al., "A miniaturized LTCC low -pass filter based on the lumped circuit model," in Proc. International Conference on Microwave and Millimeter Wave Technology (ICMMT), 2010, pp. 1578-1580. 
[3] Y. S. Dai and X. Z. Chen, "LTCC multistage structure for high performance micro research of bandpass filter," The Modern Electronic Technology, 2014.

[4] Y. S. Dai, B. S. Li, Z. H. Ye, D. L. Lu, F. Wang, and Z. D. Song, "A miniaturized LTCC bandpass filter with low insertion loss and high image rejection within 6.5 to $7.1 \mathrm{GHz}$ frequency range," in Proc. SINGAPORE: Asia Pacific Microwave Conference, 2009, pp. 1307-1309.

[5] B. S. Li, "Sideband steep LTCC filter design and research," Nanjing University of Science and Technology, 2007.

[6] X. H. Huang and G. A. Wu, "Multiple transmission zeros LTCC band pass filter," The Design and Implementation of Semiconductor Technology, vol. 4, no. 12, pp. 957-961, 2011.

[7] C. Q. Scrantom and J. C. Lawson, "LTCC technology: Where we are and where we're going," in Proc. IEEE MTT-S Int. Microwave Symp. Dig., 1999, pp. 193-200.

[8] Y. S. Dai, Y. F. Yao, B. S. Li, D. L. Lu, Y. Yuan, and S. L. Xiao, "Design and implementation of an LTCC filter with high stopband rejection," in Proc. SINGAPORE: IEEE International Symposium on Radio Frequency Integration Technology, 2009, pp. 51-54.

[9] L. J. Wang, "LTCC ultra-wideband filter research and design," Nanjing University of Science and Technology, 2007.

[10] D. M. Pozar, Microwave Engineering, 3rd ed. Beijing: Publishing House of Electronics Industry, 2006.

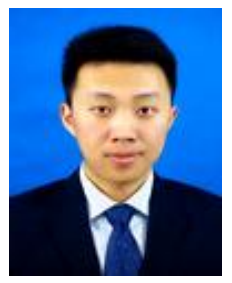

Bowen Li was born in 1990 in China. He is currently doing his master degree, of which the main research direction is microwave circuit.

$\mathrm{He}$ is with Nanjing University of Science and Technology, majoring in electronic and communication engineering, his research results include 2 invention patents.

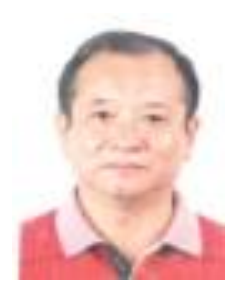

Yongsheng Dai was born in 1956 in China. He got the master degree on direction of microwave millimeter wave integrated circuits, microwave subsystem, antenna array, etc.

He is currently a professor of Nanjing University of Science and Technology. His research results include more than 50 invention patents. He has published over 90 papers in various international and national academic conferences and various professional journals, over 30 papers in international conferences and journal, including 8 Asia Pacific microwave meeting. He is also working as a senior technology consultant in many companies.

Prof. Dai is a senior member of IEEE, reviewers of the IEEE Transactions on Microwave Theory and Techniques (T-MTT), Asia-Pacific International Conference on Microwave (APMC2005) and Asia-Pacific International Conference on Microwave (APMC2002), South Korea ETRI (Electronic Telecommunications Research Journal ), etc. 

Control and Automation Engineering 
